\title{
$r$-Domination Problems on Homogeneously Orderable Graphs
}

\author{
Feodor F. Dragan, ${ }^{1}$ Falk Nicolai ${ }^{2}$ \\ ${ }^{1}$ Department of Mathematics and Cybernetics, Moldova State University, A. Mateevici str. 60, \\ Chişinău 2009, Moldova \\ ${ }^{2}$ Gerhard-Mercator-Universität-GH-Duisburg, FB Mathematik, FG Informatik, D 47048 \\ Duisburg, Germany
}

Received 27 March 1995; accepted 25 January 1997

\begin{abstract}
In this paper, we consider $r$-dominating cliques in homogeneously orderable graphs (a common generalization of dually chordal and distance-hereditary graphs) and their relation to strict $r$-packing sets. We prove that a homogeneously orderable graph $G$ possesses an $r$-dominating clique if and only if for any pair of vertices $x, y$ of $G d(x, y) \leq r(x)+r(y)+1$ holds where $r: V \rightarrow \mathbb{N}$ is a given vertex function. Furthermore, we show that for homogeneously orderable graphs with $r$-dominating cliques the cardinality of a maximum strict $r$-packing set equals the cardinality of a minimum $r$-dominating clique provided the last parameter is not two. Finally, we present two efficient algorithms: The first one decides whether a given homogeneously orderable graph has an $r$-dominating clique and, if so, computes both a minimum $r$-dominating clique and a maximum strict $r$-packing set of the graph. The second one computes a minimum connected $r$-dominating set in a homogeneously orderable graph. (C) 1997 John Wiley \& Sons, Inc. Networks 30: 121-131, 1997
\end{abstract}

\section{INTRODUCTION}

In a graph $G=(V, E)$, a subset $D \subseteq V$ is a dominating set iff each vertex $v \in V \backslash D$ has at least one neighbor in $D$. Often certain constraints for dominating sets are required: The dominating set must be connected (connected dominating set), complete (dominating clique), independent (independent dominating set), and so on.

Since $V$ itself is a dominating set of $G$, every graph

Correspondence to: F. Nicolai; e-mail: nicolai@informatik.uniduisburg.de

An extended abstract of this paper was presented at the Workshop on "Fundamentals of Computation Theory" FCT'95, Dresden, Germany (cf. [13])

Contract grant sponsors: DAAD; DFG has a dominating set, but computing a minimum one (i.e., a dominating set of smallest size) is, in general, an NP. hard problem. For special graph classes, the situation is sometimes much better. There are many papers concerned with finding minimum dominating sets in special graphs - for a bibliography of domination, cf. [18], for a compact survey of special graph classes, we refer to $[3]$.

Among the variations of the dominating set problem, the dominating clique one is of a somewhat different nature since not every graph has a dominating clique. Indeed, there are two problems - first, decide whether a given graph possesses a dominating clique (this we will call the decision problem) and, if so, then compute a minimum one (the minimality problem). For the wellknown class of weakly chordal graphs (i.e., those graphs 
which do not contain an induced cycle or its complement of length larger than four), the decision problem is $\mathbb{N P}$ complete (cf. [8]). In chordal graphs (the weakly chordal graphs which do not contain an induced 4-cycle), the decision problem is easy but it is an $\mathbb{N P}$-hard problem to compute a minimum dominating clique (cf. [20]). In contrast, there is a polynomial time algorithm to compute a minimum dominating clique in strongly chordal graphs (cf. [19]), an important subclass of chordal graphs.

In this paper, we investigate the more general problem of $r$-domination. Given a graph $G$ and a vertex function $r: V(G) \rightarrow \mathbb{N}$ (i.e., a nonnegative integer is assigned to each vertex), a set $D \subseteq V r$-dominates $G$ (is an $r$-dominating set) iff for each vertex $v$ of $V \backslash D$ there is a vertex $x$ in $D$ such that $d_{G}(v, x) \leq r(v)$, where $d_{G}$ is, as usual, the distance metric on $G$. Obviously, with $r(v)=1$ for all $v \in V$, the classical domination problem is a special case of the $r$-domination problem. Again, certain constraints for an $r$-dominating set are considered, yielding the problems $r$-dominating clique, connected $r$-dominating set, and so on.

Note that the connected $r$-dominating set problem is a generalization of the Steiner tree problem (cf. [10]). Indeed, given a Steiner set $T$, we assign to each vertex $t$ $\in T$ the value $r(t):=0$ (for all other vertices $v$ define $r(v$ ) $:=|V(G)|)$ and then compute a minimum connected $r$ dominating set which is a Steiner tree. In [7], we already presented a quadratic time algorithm for the Steiner tree problem on homogeneously orderable graphs. Note that the $r$-dominating clique problem is a generalization of the central vertex (a vertex with minimal eccentricity) problem (cf. [11]).

In [12], the existence criterion for chordal graphs given in [20] is generalized in terms of $r$-dominating cliques and is proved to be valid (in this generalized form) for Helly graphs and chordal graphs. Again, the computation of a minimum $r$-dominating clique is an $\mathbb{N P}$-hard problem for Helly graphs. For dually chordal graphs - a subclass of Helly graphs containing all strongly chordal graphs (for a characterization cf. [6, 14]) - a linear time algorithm is presented (see also [5]). In distance-hereditary graphs (cf. [1, 16, 21]), both the decision and the minimality problem can be solved in linear time as shown in [11].

In this paper, we consider $r$-dominating cliques in homogeneously orderable graphs and their relation to strict $r$-packing sets. Homogeneously orderable graphs were introduced in [7] as a common generalization of dually chordal and distance-hereditary graphs. Figure 1 presents the containment of the mentioned graph classes.

We prove that a homogeneously orderable graph $G$ possesses an $r$-dominating clique if and only if for any pair of vertices $x, y$ of $G d(x, y) \leq r(x)+r(y)+1$ holds where $r: V \rightarrow \mathbb{N}$ is a given vertex function. Again, this result is a generalization of the one for dually chordal (cf. [12]) and distance-hereditary graphs (cf. [11]). Furthermore, we show that for homogeneously orderable graphs with $r$-dominating cliques the cardinality of a maximum strict $r$-packing set equals the cardinality of a minimum $r$-dominating clique provided that the last parameter is not two.

Finally, we present two efficient algorithms which run in quadratic time if an $h$-extremal ordering is given. The first one decides whether a given homogeneously orderable graph has an $r$-dominating clique and, if so, computes both a minimum $r$-dominating clique and a maximum strict $r$-packing set of the graph. The second one computes a minimum connected $r$-dominating set in homogeneously orderable graphs. An $h$-extremal ordering of a given homogeneously orderable graph can be computed in time $O\left(n^{3}\right)$ [7]. For corresponding algorithms for dually chordal graphs, we refer to $[5,10]$; for distance-hereditary graphs, see $[4,9,11]$.

The following table summarizes these algorithmic results. Hereby, $n$ is the number of vertices and $m$ is the number of edges of a graph:

\begin{tabular}{|c|c|c|c|c|}
\hline \multirow[b]{2}{*}{ Class } & \multirow[b]{2}{*}{ Recognition } & \multicolumn{2}{|c|}{$r$-Dominating Clique } & \multirow{2}{*}{$\begin{array}{l}\text { Minimum Connected } \\
r \text {-Dominating Set }\end{array}$} \\
\hline & & Decision & Minimum & \\
\hline Trees & $O(n)$ folk & \multicolumn{2}{|c|}{$O(n)$ folk } & $O(n)$ folk \\
\hline Chordal graphs & $O(n+m)[15]$ & $O(n m)[20]$ & $\mathbb{N P}[18]$ & $\mathbb{N P}[18]$ \\
\hline Distance-hereditary graphs & $O(n+m)[16]$ & \multicolumn{2}{|c|}{$O(n+m)[11]$} & $O(n+m)[4]$ \\
\hline Dually chordal graphs & $O(n+m)[6,10]$ & \multicolumn{2}{|c|}{$O(n+m)[5,12]$} & $O\left(n^{2}\right)[5,10]$ \\
\hline Weakly chordal graphs & $O\left(m n^{2}\right)[22]$ & \multicolumn{2}{|c|}{$\mathbb{N P}[8]$} & $\mathbb{N P}[18]$ \\
\hline Homogeneously orderable graphs & $O\left(n^{3}\right)[7]$ & \multicolumn{2}{|c|}{$O\left(n^{2}\right)^{*}$ here } & $O\left(n^{2}\right)^{*}$ here \\
\hline
\end{tabular}

* An $h$-extremal ordering must be given as input. 


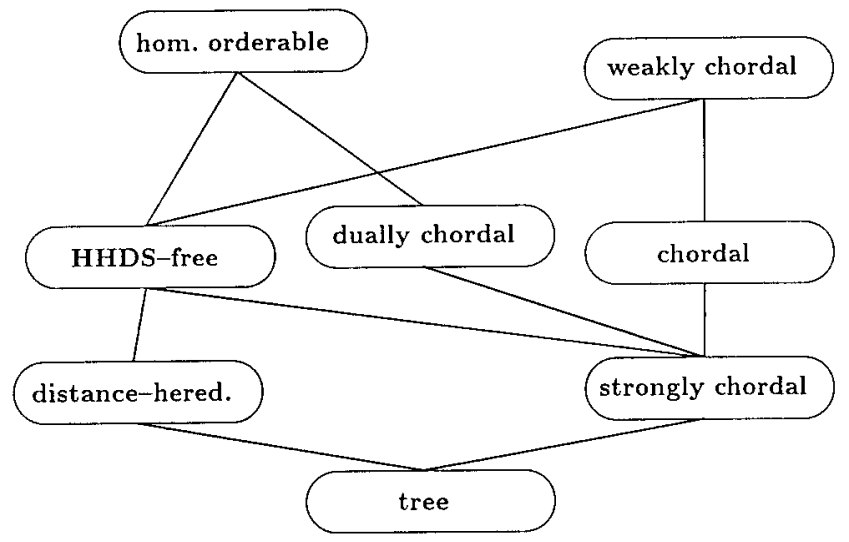

Fig. 1. Containment of graph classes.

\section{PRELIMINARIES}

Throughout this paper, all graphs $G=(V, E)$ are finite, undirected, simple (i.e., loop-free and without multiple edges) and connected.

A path is a sequence of vertices $v_{0}, \ldots, v_{k}$ such that $v_{i} v_{i+1} \in E$ for $i=0, \ldots, k-1$; its length is $k$. The distance $d_{G}(u, v)$ of vertices $u, v$ is the minimal length of any path connecting these vertices. Obviously, $d_{G}$ is a metric on $G$. If no confusion can arise, we will omit the index $G$. For a set $S \subseteq V$ and a vertex $v \in V$, we define the distance of $v$ to $S$ as

$$
d(v, S):=\min \{d(v, x): x \in S\}
$$

Let $e(v)$ denote the eccentricity of vertex $v \in V$ :

$$
e(v):=\max \{d(v, u): u \in V\}
$$

Then, the radius $\operatorname{rad}(G)$ of $G$ is the minimum over all eccentricities $e(v), v \in V$, whereas the diameter $\operatorname{diam}(G)$ of $G$ is the maximum over all eccentricities $e(v)$ for $v$ in $V$.

The $k$-th neighborhood $N^{k}(v)$ of a vertex $v$ of $G$ is the set of all vertices of distance $k$ to $v$ :

$$
N^{k}(v):=\left\{u \in V: d_{G}(u, v)=k\right\}
$$

Instead of $N^{1}(v)$, we will write $N(v)$ for the (first, open) neighborhood of $v$. For a vertex set $U \subseteq V$, let

$$
N(U):=\bigcup_{u \in U} N(u) \backslash U
$$

The disk of radius $k$ centered at $v$ is the set of all vertices of distance at most $k$ to $v$ :

$$
D(v, k):=\left\{u \in V: d_{G}(u, v) \leq k\right\}=\bigcup_{i=0}^{k} N^{i}(v)
$$

Analogously to neighborhoods of sets, we define for $U$ $\subseteq V$

$$
D(U, k):=\bigcup_{u \in U} D(u, k)
$$

A nonempty set $H \subseteq V$ is homogeneous in $G=(V, E)$ iff all vertices of $H$ have the same neighborhood in $V \backslash H$ :

$$
N(u) \cap(V \backslash H)=N(v) \cap(V \backslash H) \text { for all } u, v \in H,
$$

i.e., any vertex $w \in V \backslash H$ is adjacent to either all or none of the vertices from $H$.

A homogeneous set $H$ is proper iff $|H|<|V|$. Trivially for each $v \in V$, the singleton $\{v\}$ is a proper homogeneous set. Note also that for a subset $U \subset V$ if a set $H$ $\subseteq U$ is homogeneous in $G$ then it is homogeneous also in the induced subgraph $G(U)$ but not vice versa.

In the sequel, a subset $U$ of $V$ is a $k$-set iff $U$ induces a clique in the power $G^{k}$, i.e., for any pair $x, y$ of vertices of $U$ we have $d_{G}(x, y) \leq k$.

Let $U_{1}, U_{2}$ be disjoint subsets of $V$. If every vertex of $U_{1}$ is adjacent to every vertex of $U_{2}$, then $U_{1}$ and $U_{2}$ form a join, denoted by $U_{1} \bowtie U_{2}$. A set $U \subseteq V$ is join-splitted iff $U$ can be partitioned into two nonempty sets $U_{1}, U_{2}$ such that $U=U_{1} \bowtie U_{2}$.

Next, we recall the definition of homogeneously orderable graphs as given in [7]: A vertex $v$ of $G=(V$, $E$ ) with $|V|>1$ is h-extremal iff there is a proper subset $H \subset D(v, 2)$ which is homogeneous in $G$ and for which $D(v, 2) \subseteq D(H, 1)$ holds, i.e., $H$ dominates $D(v, 2)$. Thus, the sets $H$ and $D(v, 2) \backslash H$ form a join.

A sequence $\sigma=\left(v_{1}, \ldots, v_{n}\right)$ is an $h$-extremal ordering iff for any $i=1, \ldots, n-1$ the vertex $v_{i}$ is $h$-extremal in $G_{i}:=G\left(\left\{v_{i}, \ldots, v_{n}\right\}\right)$. A graph $G$ is homogeneously orderable iff $G$ has an $h$-extremal ordering.

In [7], it is proved that a graph is homogeneously orderable if and only if the hypergraph of the maximal join-split sets is a dual hypertree if and only if the square $G^{2}$ of $G$ is chordal and each maximal 2-set of $G$ is joinsplit.

This local structure of homogeneously orderable graphs implies a simple recognition algorithm using the chordality of the square of $G$.

Theorem 2.1 ([7]). Homogeneously orderable graphs can be recognized in cubic time $O\left(n^{3}\right)$. An h-extremal ordering can be computed within the same time bound.

Furthermore, in [7], a characterization of hereditary homogeneously orderable graphs (i.e., those graphs for 
which every induced subgraph is also homogeneously orderable) is given. They are exactly the house-hole-domino-sun-free graphs (HHDS-free graphs).

From characterizations given in [5] and [21], we immediately obtain that both dually chordal and distancehereditary graphs are homogeneously orderable graphs.

The following two lemmata are important for the sequel:

Lemma 2.2 ([7]). For any graph $G$ and any h-extremal vertex $v$ of $G$ with $e(v) \geq 2$, there is a homogeneous set $H \subseteq N(v)$ dominating $D(v, 2)$.

Lemma 2.3 ([7]). If $G$ is a homogeneously orderable graph and $v$ is an h-extremal vertex of $G$ with $e(v) \geq 2$, then $G \backslash\{v\}$ is an isometric subgraph of $G$.

Hereby, a (connected) induced subgraph $F$ of $G$ is isometric iff the distances within $F$ are the same as in $G$, i.e., for any pair of vertices $x, y$ of $F$ we have $d_{F}(x, y)$ $=d_{G}(x, y)$.

Finally, we recall the concept of $r$-domination. For a vertex function $r: V \rightarrow \mathbb{N}$ (note that we assume zero to be a natural number), a set $D \subseteq V r$-dominates a subset $U \subseteq V$ iff for each vertex $u \in U \backslash D$ there is a vertex $x$ $\in D$ such that $d_{G}(u, x) \leq r(u)$ holds. If $U=V$, then $D$ is an $r$-dominating set for $G$.

An $r$-dominating set $D$ is minimal iff for any vertex $x$ $\in D$ the set $D \backslash\{x\}$ does not $r$-dominate $G$. A minimal $r$-dominating set $D$ is minimum iff $D$ has the smallest cardinality among all minimal $r$-dominating sets of $G$. Analogously, one can define connected $r$-dominating sets and $r$-dominating cliques.

If $C$ is a minimal $r$-dominating clique of a graph $G$, then the minimality of $C$ implies that for every vertex $c$ of $C$ there must be a vertex $x_{c}$ in $G$ such that $d\left(x_{c}, c\right)$ $\leq r\left(x_{c}\right)$ and $d\left(x_{c}, c^{\prime}\right)>r\left(x_{c}\right)$ for all $c^{\prime} \in C \backslash\{c\}$, i.e., $x_{c}$ is $r$-dominated only by $c$. Such a vertex $x_{c}$ we call a private neighbor of $c$.

A dual concept is the following: A set $S \subseteq V$ is called strict r-packing set iff for all vertices $x, y$ of $S$ the equation $d(x, y)=r(x)+r(y)+1$ holds. As above, we can define maximal and maximum strict $r$-packing sets.

So, we have the following parameters:

- $\pi_{r}(G)$ - the size of a maximum strict $r$-packing set of $G$,

- $\gamma_{r}(G)$ - the size of a minimum $r$-dominating set of $G$,

- $\gamma_{r, \text { con }}(G)$ - the size of a minimum connected $r$-dominating set of $G$ and

- $\gamma_{r, c l}(G)$-the size of a minimum $r$-dominating clique of $G$, or $\infty$ if $G$ has no $r$-dominating clique.
Note that for arbitrary graphs $G$ we obviously have

$$
\pi_{r}(G) \leq \gamma_{r}(G) \leq \gamma_{r, c o n}(G) \leq \gamma_{r, c l}(G)
$$

\section{GRAPH-THEORETIC RESULTS}

For the sequel, let $G$ be a graph with vertex function $r$ : $V \rightarrow \mathbb{N}$. Define $Z(G):=\{v \in V(G): r(v)=0\}$. By definition, any $r$-dominating set must include $Z(G)$. Thus, if $Z(G)$ does not induce a complete subgraph of $G$, then $G$ has no $r$-dominating clique.

The following straightforward lemma handles the case $e(v) \leq 1$ for some vertex $v$ of $G$. So, in the sequel, we may assume that $e(v) \geq 2$.

Lemma 3.1. If $v$ is a vertex with $e(v) \leq 1$ and $Z(G)$ is complete, then we have

1. If $Z(G) \neq \varnothing r$-dominates $G$, then $Z(G)$ is both minimum r-dominating clique and maximum strict r-packing set of $G$.

2. If $Z(G) \neq \varnothing$ does not $r$-dominate $G$, then $Z(G)$

$\cup\{v\}$ is a minimum r-dominating clique and $Z(G)$

$\cup\{u\}$ is a maximum strict r-packing set of $G$ where $u$ is a private neighbor of $v$.

3. If $Z(G)=\varnothing$, then $\{v\}$ is both minimum $r$-dominating clique and maximum strict r-packing set of $G$.

\subsection{The Existence of r-Dominating Cliques}

Lemma 3.2. Let $G$ be a homogeneously orderable graph with vertex function $r: V \rightarrow \mathbb{N}, v$ be an h-extremal vertex such that $e(v) \geq 2$, let $H \subseteq N(v)$ be a homogeneous set dominating $D(v, 2)$. Furthermore, let $S$ be an arbitrary subset of $V$ containing $v$ and fulfilling

$$
\forall x, y \in S: d_{G}(x, y) \leq r(x)+r(y)+1 .
$$

Define $S^{\prime}:=(S \backslash\{v\}) \cup\{w\}$, where $w$ is either

(H1) a vertex from $S \cap N(v) \cap Z(G)$ if this intersection is nonempty but $S \cap H \cap Z(G)=\varnothing$, or

(H2) a vertex from $S \cap H$ with minimal $r$-value if $H$ $\cap S \neq \varnothing$, or

(H3) a vertex from $H$ with minimal $r$-value otherwise.

Then, $S^{\prime}$ fulfills $(P)$ in $G^{\prime}:=G \backslash\{v\}$ with respect to $r^{\prime}$, where $r^{\prime}(x):=r(x)$ for all $x \in V \backslash\{w, v\}$ and 
$r^{\prime}(w)$

$$
:=\left\{\begin{array}{lll}
0 & :(H 1) \text { or } r(v)=0, \\
\min \{r(w), r(v)-1\} & : & (H 2) \text { and } r(v) \geq 1, \\
r(v)-1 & : & (H 3) \text { and } r(v) \geq 1 .
\end{array}\right.
$$

Proof. Suppose the contrary, i.e., there are vertices $x$, $y \in S^{\prime}$ such that $d(x, y)>r^{\prime}(x)+r^{\prime}(y)+1$ (recall that by Lemma $2.3 G^{\prime}$ is an isometric subgraph of $G$ ). Obviously, one of these vertices must be $w$, say $x=w$, and $r^{\prime}(w) \neq r(w)$. Thus, we are either in case $(H 2)$ or (H3) and, hence, $w \in H$. Furthermore, $d(w, y)>r(v)$ $+r(y)$ if $r(v)>0$ and $d(w, y)>r(y)+1$ otherwise, i.e., we have $d(w, y) \geq 2$.

First assume that $d(v, y) \geq 2$. Since $v$ is $h$-extremal and the homogeneous set $H \subseteq N(v)$ dominates $D(v, 2)$, we have $d(v, y)=d(w, y)+1$. But, then,

$$
d(v, y)=d(w, y)+1>r(v)+r(y)+1,
$$

contradicting the assumption that $S$ fulfills $(P)$.

Next assume that $y \in N(v)$. We immediately conclude that $d(w, y)=2$ and $r(y)=0$. Hence, $y \in H \cap S$ and case (H3) is not possible. From the choice of $w$ [in case (H2)], we obtain $w \in H \cap S$ and $r(w)=0$. Thus, we have two nonadjacent vertices in $S$ both having $r$-value zero, a contradiction.

Theorem 3.3. For any homogeneously orderable graph $G$ with vertex function $r: V \rightarrow \mathbb{N}$ and for any subset $S$ of $V$, we have that

$S$ is $r$-dominated by some clique $C$ of $G$ if and only if $d_{G}(x, y) \leq r(x)+r(y)+1$ for all $x, y \in S$.

Proof. Obviously, if $S$ is $r$-dominated by some clique $C$, then the distance requirements are fulfilled. The converse we prove by induction on the size of $G$. Let $v$ be an $h$-extremal vertex. We may assume that $e(v) \geq 2$ by Lemma 3.1. Hence, we can choose a homogeneous set $H$ $\subseteq N(v)$ dominating $D(v, 2)$. Let $S$ be an arbitrary subset of $V$ which fulfills the distance requirements. If $v \notin S$, then we are done by the induction hypothesis and Lemma 2.3. So, let $v \in S, G^{\prime}:=G \backslash\{v\}$ and $S^{\prime}:=(S \backslash\{v\})$ $\cup\{w\}$, where $w \in N(v)$ is chosen according to the rules $(H 1)-(H 3)$ of Lemma 3.2. By Lemma 3.2 and the induction hypothesis, $S^{\prime}$ is $r^{\prime}$-dominated $\left(r^{\prime}\right.$ defined as in Lemma 3.2) by some clique $C^{\prime}$ in $G^{\prime}$. If $r(v) \geq 1$, we are done since in all cases the vertex $r^{\prime}$-dominating $w r$-dominates $v$, too. If $r(v)=0$, we have $r^{\prime}(w)=0$ and, hence, $C^{\prime} \cap N(v)$ is nonempty. Suppose that $C$ $:=\{v\} \cup\left(C^{\prime} \cap N(v)\right)$ does not $r$-dominate $S$ in $G$. Since $C^{\prime}$ is an $r^{\prime}$-dominating clique for $S^{\prime}$ in $G^{\prime}$, there must be vertices $x \in C^{\prime} \cap N^{2}(v)$ and $y \in S$ such that $d(x, y)=r(y)$ and $d(y, C)>r(y)$. Note that $r(y) \geq 1$ since $r(x)>0$ and $x \neq y$. Thus, $y \notin N(v)$, since, otherwise, $v r$-dominates $y$.

CAses $(H 2)$ and $(H 3)$. Here, we have $w \in H$. Since $r^{\prime}(w)=0$ implies that $w \in C$, we obtain $d(w, y)$ $\geq r(y)+1$. From $d(v, y)=d(w, y)+1$ and $r(v)$ $=0$, we conclude that $d(v, y) \geq r(v)+r(y)+2$, a contradiction.

CASE $(H 1)$. We immediately conclude that $r^{\prime}(w)$ $=r(w)=0$ and $w \in N(v) \backslash H$. Thus, $d(w, y)=d(v$, $y)=r(y)+1$. If $C \cap H$ is nonempty, then choose any vertex $h \in C \cap H$ and proceed as in the above case. If $C \cap H=\varnothing$, the above considerations imply that the new clique $C:=\{v, h\} \cup\left(C^{\prime} \cap N(v)\right)$, with $h \in H$, is an $r$-dominating clique in $G$.

With $r(v)=1$ for all $v \in V$, we immediately conclude that

Corollary 3.4. A homogeneously orderable graph $G$ is dominated by some clique if and only if $\operatorname{diam}(G) \leq 3$.

Corollary 3.5. For homogeneously orderable graphs $G$, we have $2 \operatorname{rad}(G) \geq \operatorname{diam}(G) \geq 2(\operatorname{rad}(G)-1)$.

Proof. Suppose that $\operatorname{diam}(G)<2(\operatorname{rad}(G)-1)$. Then, by Theorem 3.3 for $r(v):=\operatorname{rad}(G)-2, v \in V$, there exists an $r$-dominating clique $C$ in $G$. Hence, any vertex $v$ of $G$ has $e(v) \leq \operatorname{rad}(G)-1$, a contradiction to the definition of the radius.

\subsection{Minimum $r$-Dominating Cliques and Maximum Strict $r$-Packing Sets}

Here, we consider the relationship of the parameters $\pi_{r}(G), \gamma_{r}(G), \gamma_{r, c o n}(G)$, and $\gamma_{r, c l}(G)$ for homogeneously orderable graphs with $r$-dominating cliques. Recall that

$$
\pi_{r}(G) \leq \gamma_{r}(G) \leq \gamma_{r, c o n}(G) \leq \gamma_{r, c l}(G)
$$

Lemma 3.6. Let $G$ be a homogeneously orderable graph with h-extremal vertex $v, e(v) \geq 2$ and a vertex function $r: V \rightarrow \mathbb{N}$ such that $r(v) \geq 1$. Moreover, assume that $G$ is not $r$-dominated by a single vertex but by some minimum clique containing $v$. Then, there is an r-dominating clique of $G$ of the same size which does not contain $v$.

Proof. Let $C$ be a minimum $r$-dominating clique of $G$ containing $v$. Thus, $C \subseteq D(v, 1)$. Since $v$ is $h$-extremal and $e(v) \geq 2$, we can choose a homogeneous set $H \subseteq N(v)$ dominating $D(v, 2)$. If $C \cap H$ is empty, we can replace $v$ in $C$ by any vertex from $H$. Otherwise, the minimality 
of $C$ immediately implies that $C \backslash\{v\} \subseteq H$. Thus, we can replace $v$ by some vertex $w$ from $N^{2}(v)$.

In the sequel, we will often apply Theorem 3.3 and Lemma 3.2. In all of these cases, we will have $S$ $:=V(G)$. Thus, rule $(H 3)$ for the choice of $w$ will never be used.

Lemma 3.7. Let $G$ be a homogeneously orderable graph which is r-dominated by some clique but not by a single vertex, i.e., $1<\gamma_{r, c l}(G)<\infty$. Let $v$ be any h-extremal vertex of $G$ with $e(v) \geq 2$ and $r(v) \geq 1$. Furthermore, let $H \subseteq N(v)$ be a homogeneous set dominating $D(v, 2)$. Define $w, G^{\prime}$, and $r^{\prime}$ as in Lemma 3.2 with $S:=V(G)$. Then, any $r^{\prime}$-dominating clique $C^{\prime}$ in $G^{\prime}$ is an r-dominating clique in $G$, and if $C$ is a minimum $r$-dominating clique in $G$, then there exists an $r^{\prime}$-dominating clique $C^{\prime}$ in $G^{\prime}$ of the same size, i.e.,

$$
\gamma_{r, c l}(G)=\gamma_{r^{\prime}, c l}\left(G^{\prime}\right)
$$

Proof. Note at first that the rules $(H 1)$ and $(H 2)$ of Lemma 3.2 immediately imply that $v$ is $r$-dominated by the vertex $r^{\prime}$-dominating $w$. Thus, any $r^{\prime}$-dominating clique $C^{\prime}$ in $G^{\prime}$ is an $r$-dominating clique in $G$ and, hence, $\gamma_{r, c l}(G) \leq \gamma_{r^{\prime}, c l}\left(G^{\prime}\right)$. To prove that $\gamma_{r, c l}(G) \geq \gamma_{r^{\prime}, c l}\left(G^{\prime}\right)$, let $C$ be any minimum $r$-dominating clique of $G$. By Lemma 3.6, we may assume that $v \notin C$. If $C r^{\prime}$-dominates $G^{\prime}$, then we are done. So, assume that $C$ does not $r^{\prime}$ dominate $G^{\prime}$, i.e., there is a vertex $x$ in $G^{\prime}$ such that $d(x$, $C)>r^{\prime}(x)$. But $C$ is an $r$-dominating clique in $G$, i.e., $d(x, C) \leq r(x)$. From the definition of $r^{\prime}$, we conclude that $x=w$ and $r(w) \neq r^{\prime}(w)=r(v)-1$, i.e., $w$ is chosen by rule $(H 2)$.

Assume that $r(v) \geq 2$. Then, $d(w, C) \geq r(v) \geq 2$ implies that either $C \subseteq H$ or $C \cap D(v, 2)=\varnothing$. In the latter case, we obtain the contradiction $d(v, C)=d(w$, $C)+1 \geq r(v)+1$. Thus, $C \subseteq H$ and $d(w, C)=2$. Rule (H2) together with $r(w) \geq r(v) \geq 2$ implies that for all vertices $h \in H$ we have $r(h) \geq 2$. We claim that $|C|$ $=1$, i.e., $G$ is $r$-dominated by a single vertex, which is a contradiction. Suppose that there are vertices $c_{1}, c_{2}$ in $C$. Let $p_{1}, p_{2}$ be private neighbors of these vertices, i.e., $d\left(c_{i}, p_{i}\right)=r\left(p_{i}\right), i=1,2$, and $d\left(c_{i}, p_{j}\right)=r\left(p_{j}\right)+1$, $i \neq j$. Since $c_{1}, c_{2}$ are also in the homogeneous set $H$, the private neighbors must belong to $H$. But this implies that $r\left(p_{i}\right) \leq 1, i=1,2$, a contradiction.

So, $r(v)=1$. Then, $C$ contains at least one vertex from $N(v)$. From the choice of $w$ according to $(H 2)$ and since $w \notin C$, we obtain $r(z) \geq 1$ for all vertices $z \in N(v)$ and $r^{\prime}(w)=0$. If $C \subseteq H$, then $\{w, u\}$ is an $r^{\prime}$-dominating clique in $G^{\prime}$, where $u$ is an arbitrary vertex from $N(v) \backslash H$ or from $N^{2}(v)$ if $H=N(v)$. Now assume that $C \nsubseteq H$. If $C \cap H=\varnothing$, then replace some vertex of $N(v) \cap C$ (recall that their $r$-values are at least one) by $w$. Otherwise, $C^{\prime}$ $:=(C \backslash H) \cup\{w\}$ is an $r^{\prime}$-dominating clique in $G^{\prime}$.

Consequently, for each minimum $r$-dominating clique $C$ of $G$, there is an $r^{\prime}$-dominating clique $C^{\prime}$ in $G^{\prime}$ of the same size as $C$. So, we are done.

Theorem 3.8. If a homogeneously orderable graph $G$ possesses an $r$-dominating clique and $\gamma_{r, c l}(G) \neq 2$, then $\pi_{r}(G)=\gamma_{r, c l}(G)$.

Proof. Since for $\gamma_{r, c l}(G)=1$ there is nothing to show, let $\gamma_{r, c l}(G) \geq 3$. Let $v$ be an $h$-extremal vertex of $G$. If $e(v)=1$, then we are done by Lemma 3.1. So, assume that $e(v) \geq 2$ and let $H \subseteq N(v)$ be a homogeneous set dominating $D(v, 2)$. First, we consider the case $r(v)=0$. If $Z(G) r$-dominates $G$, then it is both a minimum $r$ dominating clique and a maximum strict $r$-packing set of $G$. Otherwise, we show that $C:=Z(G) \cup\{h\}$ with $h$ $\in H$ is an $r$-dominating clique in $G$. Assume that $Z(G)$ $\cap H \neq \varnothing$ and let $h^{\prime}$ be a vertex from this intersection. We prove that $Z(G) r$-dominates $G$. Suppose for the contrary that there is a vertex $x$ with $d(x, Z(G))>r(x)$. Obviously, $x \notin D(v, 1)$, implying that $d(v, x)=d\left(h^{\prime}\right.$, $x)+1$. Consequently, $d(x, v)>r(x)+r(v)+1$, a contradiction to Theorem 3.3. Thus, $Z(G) \cap H$ is empty and $C$ is complete. By similar arguments (replace $h^{\prime}$ by $h), C r$-dominates $G$. Since $Z(G)$ does not $r$-dominate $G$, the clique $C$ is minimum and there is a private neighbor $x$ of $h$. Thus, $Z(G) \cup\{x\}$ is a maximum strict $r$-packing set of $G$. This settles the case $r(v)=0$.

To prove the assertion for $r(v) \geq 1$, we proceed by induction on the size of $G$. Define $w, G^{\prime}$, and $r^{\prime}$ as in Lemma 3.2 with $S:=V$ [thus, case $(H 3)$ cannot arise]. From Lemma 3.7, we have $\gamma_{r^{\prime}, c l}\left(G^{\prime}\right)=\gamma_{r, c l}(G)$. By the induction hypothesis, we have that $\pi_{r^{\prime}}\left(G^{\prime}\right)=\gamma_{r^{\prime}, c l}\left(G^{\prime}\right)$. Since $\pi_{r}(G) \leq \gamma_{r, c l}(G)$, it remains to show that $\pi_{r^{\prime}}\left(G^{\prime}\right)$ $\leq \pi_{r}(G)$.

By Lemma 2.3, we have only to consider the case $r^{\prime}(w)=r(v)-1<r(w)$ and $w$ belongs to a maximum strict $r^{\prime}$-packing set $P^{\prime}$ of $G^{\prime}$. Since we only changed the radius of $w$, we have $d(w, y) \neq r(w)+r(y)+1$ for all vertices $y \in P^{\prime} \backslash\{w\}$.

Suppose that there is a vertex $y \in N(v) \cap P^{\prime} \backslash\{w\}$. From $d(w, y)=r(v)+r(y) \leq 2, r(v) \geq 1$, and the choice of $w$ in case (H2) of Lemma 3.2, we conclude that $d(w, y)=2, y \in H, r(y)=r(w)=r(v)=1$. Assume that there is a vertex $x$ of $P^{\prime} \backslash\{w, y\}$. Then, we get $d(x, y)=2+r(x)$ and $d(w, x)=1+r(x)$. Since $H$ is homogeneous and $w, y$ are in $H$, we conclude that $x \in H$. But then $r(x)=0$, which is impossible in case $(H 2)$. Thus, $\pi_{r^{\prime}}\left(G^{\prime}\right)=2$, contradicting $\pi_{r^{\prime}}\left(G^{\prime}\right)$ $=\gamma_{r^{\prime}, c l}\left(G^{\prime}\right)=\gamma_{r, c l}(G) \geq 3$.

Therefore, for all $y \in P^{\prime} \backslash\{w\}$, we have $d(v, y) \geq 2$ and $d(v, y)=d(w, y)+1=r(v)+r(y)+1$. So, the set $P:=\left(P^{\prime} \backslash\{w\}\right) \cup\{v\}$ is a strict $r$-packing set of $G$ and, hence, $\pi_{r^{\prime}}\left(G^{\prime}\right) \leq \pi_{r}(G)$. 
To verify the exception for the case $\gamma_{r, c l}(G)=2$, consider an induced 4-cycle $v_{0}-v_{1}-v_{2}-v_{3}-v_{0}$ with $r\left(v_{i}\right)$ $=1$ for all $i \in\{0, \ldots, 3\}$. Obviously, $\gamma_{r, c l}(G)=2$ and $\pi_{r}(G)=1$.

Corollary 3.9. Let $G$ be a homogeneously orderable graph possessing an r-dominating clique:

$$
\begin{aligned}
& \text { 1. If } \gamma_{r, c l}(G) \neq 2 \text { or } \pi_{r}(G)>1 \text {, then } \pi_{r}(G)=\gamma_{r}(G) \\
& =\gamma_{r, \text { con }}(G)=\gamma_{r, c l}(G) \\
& \text { 2. } \gamma_{r}(G)=\gamma_{r, c o n}(G)=\gamma_{r, c l}(G)
\end{aligned}
$$

Corollary 3.10. In a homogeneously orderable graph $G$, any set of pairwise intersecting disks has either a nonempty common intersection or there is an edge such that for each of these disks at least one vertex of the edge belongs to the disk.

Proof. Let $\Phi)=\left\{D\left(x_{i}, r_{i}\right): i=1, \ldots, k\right\}$ be a set of pairwise intersecting disks and define a vertex function $r: V \rightarrow \mathbb{N}$ by

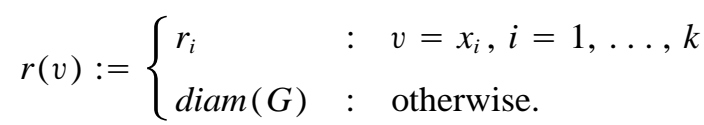

Since the disks pairwise intersect, we have $d\left(x_{i}, x_{j}\right)$ $\leq r\left(x_{i}\right)+r\left(x_{j}\right)$ and $\pi_{r}(G)=1$. Thus, by Theorem 3.3, $G$ has an $r$-dominating clique. The assertion follows from the preceding corollary.

Again, an induced 4-cycle shows that homogeneously orderable graphs are in general not Helly, i.e., it is not necessary that pairwise intersecting disks have a nonempty common intersection in a homogeneously orderable graph.

Note that Theorem 3.8 and Corollaries 3.9 and 3.10 generalize similar results for distance-hereditary graphs presented in [11].

\section{THE ALGORITHMS}

At first, we present an efficient algorithm for computing the distance matrix of certain graphs in optimal, quadratic time.

Theorem 4.1. For any graph $G$ possessing an ordering $\left(v_{1}, \ldots, v_{n}\right)$ such that for each $i=1, \ldots, n-1$ it holds that (with $G_{1}:=G$ )

1. $G_{i+1}:=G_{(\{v i+1, \ldots, v n\})}$ is an isometric subgraph of $\mathrm{G}_{i}$

2. For a given vertex $w_{i}$ in $N_{G_{i}}\left(v_{i}\right)$ and for any vertex $x$ in $N_{G_{i}}^{j}\left(v_{i}\right), j=2, \ldots, e_{G_{i}}\left(v_{i}\right)$, there is a path of length $j$ joining $x$ and $v_{i}$ and containing $w_{i}$

the distance matrix of $G$ can be computed in optimal, quadratic time $O\left(n^{2}\right)$.

Proof. The assertion is proved by induction on $n$. Let $\left(v_{1}, \ldots, v_{n}\right)$ be an ordering of $G$ according to the presumptions. By the induction hypothesis, the distance matrix of $G_{2}:=G \backslash\left\{v_{1}\right\}$ can be computed in time $O\left(n^{2}\right)$. For any vertex $x \in N\left(v_{1}\right)$, we have $d\left(v_{1}, x\right)=1$, for any vertex $x \in V \backslash D\left(v_{1}, 1\right)$, (2) implies that $d\left(v_{1}, x\right)=d\left(w_{1}\right.$, $x)+1$. For all pairs of vertices $x, y \in V \backslash\left\{v_{1}\right\}$, the distances remain the same by (1). Thus, updating the distance matrix of $G_{2}$ to $G$ takes time $O(n)$.

Note that by Lemma 2.3 any homogeneously orderable graph fulfills the presumptions of Theorem 4.1.

\section{1. $r$-Dominating Cliques and Strict $r$-Packing Sets}

By Theorem 4.1, the distance matrix of a given homogeneously orderable graph can be computed in quadratic time. Thus, by Theorem 3.3, it can be decided within the same time whether the given graph possesses an $r$ dominating clique. Moreover, by using the distance matrix, it is easy to check in quadratic time, too, whether the graph has an $r$-dominating vertex. So, assume for the sequel that a given homogeneously orderable graph $G$ is not $r$-dominated by some vertex but by some clique.

The following algorithm both computes an $r$-dominating clique of minimum size and a maximum strict $r$ packing set of $G$. It works in three rounds. In the first round, it steps through a given $h$-extremal ordering and manipulates $r$ by using the rules of Lemma 3.2 and the arguments of Lemma 3.7 until it reaches a vertex $v$ with $r(v)=0$ or $e(v)=1$. In the second one, a minimum $r$ dominating clique $C$ and a maximum strict $r$-packing set $P$ of the current graph is chosen according to Lemma 3.1 and to the proof of Theorem 3.8. By Lemma 3.7, the clique $C$ is also a minimum $r$-dominating clique in $G$. If $|C|=2$, then $\pi_{r}(G) \leq 2$, and a maximum strict $r$ packing set $P$ of the initial graph can be computed in quadratic time by only using the distance matrix.

If $|C| \neq 2$, to find a maximum strict $r$-packing set in $G$, the algorithm in the third round goes backward through the sequence and updates the parameter $P$ according to the arguments of the proof of Theorem 3.8.

In the sequel, an $h$-extremal ordering $\tau$ of a homogeneously orderable graph $G$ is given as sequence of pairs $\left(v_{i}, H_{i}\right), i=1, \ldots, n$, where $v_{i}$ is $h$-extremal in $G_{i}$ $=G\left(\left\{v_{i}, \ldots, v_{n}\right\}\right)$ and $H_{i} \subset D_{G_{i}}\left(v_{i}, 2\right)$ is a homogeneous set in $G_{i}$ which dominates $D_{G_{i}}\left(v_{i}, 2\right)$. This sequence of pairs can be obtained in $O\left(n^{3}\right)$ time by using the recogni- 
tion algorithm from [7]. Note that $\tau(i, 1):=v_{i}$ and $\tau(i$, 2) $:=H_{i}$ for $i=1, \ldots, n$. For convenience, we use the following abbreviations: $e_{i}(v):=e_{G_{i}}(v)$ and $N_{i}(v)$ $:=N(v) \cap V\left(G_{i}\right)$.

\section{Algorithm RDC}

Input: A homogeneously orderable graph $G$ with vertex function $r: V \rightarrow \mathbb{N}$ and an $h$-extremal ordering $\tau=\left(\left(v_{1}\right.\right.$, $\left.\left.H_{1}\right), \ldots,\left(v_{n}, H_{n}\right)\right)$ of $G$.

Output: A minimum $r$-dominating clique $C$ and a maximum strict $r$-packing set $P$, or 'No.'

(1) Compute the distance matrix $D(G)$ of $G$.

(2) if not $(\forall x, y \in V: d(x, y) \leq r(x)+r(y)+1)$ then stop ('No').

(3) if $\exists x \in V \forall y \in V \backslash\{x\}: d(x, y) \leq r(y)$ then stop $(\{x\},\{x\})$.

(* Now, $\left.1<\gamma_{r, c l}(G)<\infty *\right)$

(4) Compute $Z(G):=\{x \in V: r(x)=0\}$.

(5) $i:=1 ; v:=\tau(1,1) ; H:=\tau(1,2) ; G_{1}:=G ; Z_{1}$ $:=Z(G) ; r_{1}:=r$.

(* Round 1. *)

(6) while $\left(r_{i}(v) \geq 1\right)$ and $\left(e_{i}(v) \geq 2\right)$ do

(6.1) if $\left(H \cap Z_{i}=\varnothing\right)$ and $\left(N_{i}(v) \cap Z_{i} \neq \varnothing\right)$ then choose $w \in N_{i}(v) \cap Z_{i}$

(6.2) else choose $w \in H$ such that $r_{i}(w)=\min$ $\left\{r_{i}(h): h \in H\right\}$;

(6.3) $\quad r_{i+1}(w):=\min \left\{r_{i}(w), r_{i}(v)-1\right\}$;

(6.4) $\quad \sigma(i):=w ;($ *pointer to the neighbor $w$ of $v *$ )

(6.5) if $r_{i+1}(w)=0$ then $Z_{i+1}:=Z_{i} \cup\{w\}$ else $Z_{i+1}$ $:=Z_{i}$;

(6.6) forall $x \in V\left(G_{i}\right) \backslash\{v, w\}$ do $r_{i+1}(x):=r_{i}(x)$;

(6.7) $\quad G_{i+1}:=G_{i} \backslash\{v\} ; i:=i+1 ; v:=\tau(i, 1) ; H$ $:=\tau(i, 2)$

endwhile;

(* Round 2. *)

(7) if $e_{i}(v) \leq 1$ then

(7.1) if $Z_{i}=\varnothing$ then $C=P:=\{v\}$

(7.2) else if $Z_{i} r_{i}$-dominates $G_{i}$ then $C=P:=Z_{i}$

(7.3) else $C:=Z_{i} \cup\{v\}$;

(7.4) $\quad P:=Z_{i} \cup\{u\}, u$ private neighbor of $v$

(8) else $\left(* e_{i}(v) \geq 2\right.$ and $\left.r_{i}(v)=0 *\right)$

(8.1) if $Z_{i} r_{i}$-dominates $G_{i}$ then $C=P:=Z_{i}$

(8.2) else $C:=Z_{i} \cup\{h\}, h \in H$;

(8.3) $\quad P:=Z_{i} \cup\{x\}, x$ private neighbor of $h$

endif;

(* Round 3. *)

(9) if $|C|=2$ then compute $P$ by using $D(G)$

(10) else for $j:=i-1$ downto 1 do

(10.1) $\quad w:=\sigma(j) ; v:=\tau(j, 1)$;

(10.2) if $\left(r_{j+1}(w)<r_{j}(w)\right)$ and $(w \in P)$ then $P$

$:=(P \backslash\{w\}) \cup\{v\}$

endfor endif;

(11)

stop $(C, P)$.

We conclude

Theorem 4.2. In homogeneously orderable graphs, it can be decided in time $O\left(n^{2}\right)$ whether the given graph is $r$-dominated by some clique, provided that an h-extremal ordering is given. Moreover, if the graph has an r-dominating clique, then a minimum one and a maximum strict r-packing set can be computed in the same time.

Proof. We may assume that a given homogeneously orderable graph $G$ is not $r$-dominated by some vertex but by some clique. The three different cases arising in our algorithm are the following:

CASE 1. $e(v) \geq 2$ and $r(v) \geq 1$.

Define $w, G^{\prime}$, and $r^{\prime}$ according to the rules $(H 1)-$ (H3) of Lemma 3.2. By Lemma 3.7, we have that $\gamma_{r, c l}(G)=\gamma_{r^{\prime}, c l}\left(G^{\prime}\right)$, and each minimum $r^{\prime}$-dominating clique of $G^{\prime}$ is a minimum $r$-dominating clique of $G$.

CAse 2. $e(v)=1$.

In this case, round one is terminated. Then, the parameters $(C, P)$ of the current graph defined according to Lemma 3.1 are computed, and round three starts. This can be done in time $O\left(n^{2}\right)$ by using the distance matrix.

CASe 3. $e(v) \geq 2$ and $r(v)=0$.

In this case, round one is terminated. Since any $r$ dominating clique is contained in $D(v, 1)$, either $C$ $=P=Z(G)$ or $C=\{h\} \cup Z(G)$ and $P=Z(G)$ $\cup\{x\}$, where $h \in H$ and $x$ is a private neighbor of $h$. By the proof of Theorem 3.8, $C$ is a minimum $r$ dominating clique and $P$ is a maximum strict $r$-packing set of the current graph. By using the distance matrix, this step can be easily performed in quadratic time. Now round three starts.

Obviously, the overall running time is $O\left(n^{2}\right)$.

\subsection{Connected $r$-Dominating Sets}

Here, we extend the method described in [10] (see also [5]) for the connected $r$-dominating set problem on dually chordal graphs to the class of homogeneously orderable graphs. To prove the correctness of the next algorithm, we will need the following lemma from [7].

Lemma 4.3 ([7]). Let $G$ be a homogeneously orderable graph with h-extremal ordering $\sigma=\left(v_{1}, \ldots, v_{n}\right)$. Let $G^{\prime}$ be the graph obtained from $G$ by adding an arbitrary edge between vertices of $N\left(v_{1}\right)$. Then, $G^{\prime}$ is homoge- 
neously orderable and $\sigma$ remains an h-extremal ordering for $G^{\prime}$.

For the sequel, let $v$ be an $h$-extremal vertex with $e(v)$ $\geq 2$ and let $H \subseteq N(v)$ be a homogeneous set dominating $D(v, 2)$. Define $A:=N(v) \backslash H, r_{H}:=\min \{r(h): h$ $\in H\}$ and $r_{A}:=\min \{r(a): a \in A\}$ (if $A=\varnothing$, we put $\left.r_{A}:=\infty\right)$. Moreover, suppose that $v$ does not $r$-dominate $G$. Define $G^{\prime}:=G \backslash\{v\}$ and let $S^{\prime}$ be a minimum connected $r^{\prime}$-dominating set in $G^{\prime}$ ( $r^{\prime}$ will be defined in the following cases). In what follows, we describe how we can obtain a minimum connected $r$-dominating set $S$ in $G$ from $S^{\prime}$.

CASE 1. $r(v)>0$ and $\min \left\{r_{H}, r_{A}\right\}=0$.

We define $r^{\prime}(x):=r(x)$ for all vertices $x \in V\left(G^{\prime}\right)$ and claim that $S:=S^{\prime}$ is a minimum connected $r$ dominating set in $G$. Obviously, $S$ is a connected $r$ dominating set in $G$. Suppose that $S$ is not minimum, but $D$. Since we did not change the $r$-values in $G^{\prime}$, we immediately conclude that $v \in D$. Moreover, not both of $r_{A}$ and $r_{H}$ can be zero, for, otherwise, we can delete $v$ [recall that $H$ is homogeneous and dominates $D(v, 2)]$. If $r_{A}=0$, then we can replace $v$ in $D$ by some vertex $h$ in $H$ obtaining a connected $r$-dominating set of the same size as $D$ but without $v$, a contradiction. If $r_{H}=0$, then we can replace $v$ by some vertex $w$ of $A \cup N^{2}(v)$ which yields the same contradiction. Thus, $S$ is minimum.

CASE 2. $r(v)>r_{H}$ and $\min \left\{r_{H}, r_{A}\right\}>0$.

Again, we define $r^{\prime}(x):=r(x)$ for all vertices $x$ $\in V\left(G^{\prime}\right)$ and claim that the connected $r$-dominating set $S:=S^{\prime}$ is minimum in $G$. Suppose that there is a smaller connected $r$-dominating set $D$ in $G$. Since we did not change the $r$-values in $G^{\prime}$, we immediately conclude that $v \in D$. Moreover, from $\min \left\{r_{H}, r_{A}\right\}$ $>0$, we obtain $|D \cap N(v)|=1$. W.l.o.g., we may assume that $D \cap N(v) \subseteq H$.

If $D \cap N^{2}(v) \neq \varnothing$, then we can delete $v$, a contradiction to the minimality of $D$. Thus, $D \subseteq D(v, 1)$. But now we can replace $v$ by some vertex $w$ of $N^{2}(v)$ [recall that $e(v) \geq 2$ ], obtaining a connected $r$-dominating set of the same size as $D$ but without $v$, a contradiction.

CASE 3. $1<r(v) \leq r_{H}$ and $r_{A}>0$.

Choose a vertex $h$ from $H$ such that $r(h)=r_{H}$; define $r^{\prime}(h):=r(v)-1$ and $r^{\prime}(x):=r(x)$ for all remaining vertices. We claim that the connected $r$-dominating set $S:=S^{\prime}$ is minimum in $G$. Suppose that there is a connected $r$-dominating set $D$ such that $|D|<|S|$ holds.

\section{CAse 3.1. $v \notin D$.}

From the minimality of $S^{\prime}$ in $G^{\prime}$, we immediately conclude that $D$ cannot $r^{\prime}$-dominate $G^{\prime}$. Since we have only changed the $r$-value of $h$ in $G^{\prime}$ and $D$ is a connected $r$-dominating set in $G$, the only vertex in $G^{\prime}$ which is not $r^{\prime}$-dominated by $D$ is $h$, i.e., $d(h, D)>r^{\prime}(h)=r(v)-1$. But $D r$ dominates $v$ in $G$, i.e., $d(v, D) \leq r(v)$. From $r(v) \geq 2$, we have $d(h, D) \geq 2$. If $D$ $\subseteq V(G) \backslash D(v, 2)$, then we have $d(v, D)=d(h$, $D)+1>r(v)$, a contradiction. Otherwise, we immediately conclude that $D \subset H$ and $d(h, D)$ $=r(v)=2$. From $r(h)=r_{H} \geq 2$, we obtain that $h r$-dominates $G$ and $r^{\prime}$-dominates $G^{\prime}$, yielding a contradiction.

\section{CAse 3.2. $v \in D$.}

From $\min \left\{r_{H}, r_{A}\right\}>0$, we conclude that $\mid D$ $\cap N(v) \mid=1$ (recall that $v$ does not $r$-dominate $G$ ). We may assume that $D \cap N(v)=\{h\}$. If $D \cap$ $N^{2}(v) \neq \varnothing$, then we can delete $v$, contradicting the minimality of $D$. Thus, $D \subseteq D(v, 1)$. But now we can replace $v$ by some vertex $w$ of $N^{2}(v)$, obtaining a connected $r$-dominating set of the same size as $D$ but without $v$, a contradiction by Case 3.1.

CASE 4. $r(v)=1$ and $\min \left\{r_{H}, r_{A}\right\}>0$.

We distinguish between two subcases:

CASE 4.1. There is a vertex $x$ in $N^{i}(v), i \geq 2$, such that $d(x, v) \geq r(x)+2$.

We define $r^{\prime}(h):=0$ for an arbitrary vertex $h$ of $H$ and $r^{\prime}(x):=r(x)$ for all $x \in V\left(G^{\prime}\right) \backslash\{h\}$. We prove that $S:=S^{\prime}$ is a minimum connected $r$ dominating set in $G$.

Since $d(x, v) \geq r(x)+2$ and $r(v)=1$, in any connected $r$-dominating set $D$ of $G$ there must be a vertex from $N^{2}(v)$. Moreover, such a set $D$ may not contain $v$ and its intersection with $N(v)$ is a singleton since $\min \left\{r_{H}, r_{A}\right\}>0$. Thus, we may assume that $S^{\prime} \cap N(v)=\{h\}$, which implies the correctness.

CASE 4.2. For all vertices $x \in V \backslash D(v, 1)$, we have $d(x, v) \leq r(x)+1$. If $H$ is $r$-dominated by some vertex $h \in H$, then $S:=\{h\}$. Otherwise, either there is a vertex $a \in A$ which $r$-dominates $G$ or $S$ contains at least two vertices. But, then, we may choose $S:=\{v, h\}$ where $h$ is an arbitrary vertex from $H$.

CASE 5. $r(v)=0$.

If there is a vertex $w \in N(v)$ with $r(w)=0$, then we do not change the $r$-values. Otherwise, define $r^{\prime}(w)$ $:=0$ for an arbitrary vertex $w$ of $H$ and $r^{\prime}(x):=r(x)$ for all other vertices. In both cases, add the edges between each vertex of $N(v) \backslash\{w\}$ and $w$ in $G^{\prime}$ (by Lemma $4.3, G^{\prime}$ has the same $h$-extremal ordering as $G)$. Moreover, the distance matrix of $G^{\prime}$ can be obtained in linear time from the distance matrix of $G$. 
It is easy to see that $S:=S^{\prime} \cup\{v\}$ is a connected $r$-dominating set in $G$. Using the same arguments as in the preceding cases, we can prove that $S$ is a minimum one.

Finally, consider the case $e(v) \leq 1$. If there are vertices with $r$-value zero, i.e., $Z(G) \neq \varnothing$, then $Z(G)$ or $Z(G)$ $\cup\{v\}$ is a minimum connected $r$-dominating set of $G$. Otherwise, $v r$-dominates $G$.

In the following algorithm, we will use the same notions as in the algorithm RDC.

\section{Algorithm CRDS.}

Input: A homogeneously orderable graph $G$ with vertex function $r: V \rightarrow \mathbb{N}$ and an $h$-extremal ordering $\tau=\left(\left(v_{1}\right.\right.$, $\left.\left.H_{1}\right), \ldots,\left(v_{n}, H_{n}\right)\right)$ of $G$.

Output: A minimum connected $r$-dominating set $S$.

(1) Compute the distance matrix $D(G)$ of $G$.

(2) $S:=$ LocalCRDS $(1, G, r, D(G))$;

(3) $\operatorname{stop}(S)$.

Hereby, we use the following:

subroutine LocalCRDS $(j, G, r, D): S$

Input: A positive integer $j$, a homogeneously orderable graph $G$ with vertex function $r$ and distance matrix $D$.

Output: A minimum connected $r$-dominating set $S$ of $G$.

(S1) $v:=\tau(j, 1) ; H:=\tau(j, 2)$;

(S2) if $e(v) \leq 1$ then

(S3) Compute $Z(G):=\{x \in V(G): r(x)=0\}$.

(S4) if $Z(G)=\varnothing$ then $\operatorname{stop}(\{v\})$

(S5) else if $Z(G)$ is connected then $\operatorname{stop}(Z(G))$ (S6) else $\operatorname{stop}(Z(G) \cup\{v\})$

endif:

(* Now, $e(v) \geq 2$. *)

(S7) if $v r$-dominates $G$ then $\operatorname{stop}(\{v\})$.

(S8) $r_{H}:=\min \{r(h): h \in H\}$;

(S9) $A:=N(v) \backslash H$;

(S10) if $A=\varnothing$ then $r_{A}:=\infty$ else $r_{A}:=\min \{r(a): a$ $\in A\}$;

$(\mathrm{S} 11)$ if $\left(r(v)>r_{H}\right)$ or $\left(r(v)>0=r_{A}\right)$ then

(S12) $S:=\operatorname{Loca} 1 \mathrm{CRDS}(j+1, \quad G \backslash\{v\}, \quad r$, $D(G \backslash\{v\}))$;

(S13) $\quad \operatorname{stop}(S)$

(S14) else (* Now, $r(v) \leq r_{H}$ and, if $r(v)>0$ then $r_{A}$ $>0 . *)$
Theorem 4.4. In homogeneously orderable graphs, a minimum connected $r$-dominating set can be computed in time $O\left(n^{2}\right)$ provided that an h-extremal ordering is given.

Proof. The correctness follows from the preceding cases. To verify the time bound, recall that the distance matrix of $G$ can be computed in quadratic time by Theorem 4.1. In the subroutine LocalCRDS, all parts up to steps (S5) and (S28) run in time $O(n)$. In step (S5), the connectedness of $Z(G)$ must be checked. This step costs $O(n+m)$ time, but it will be performed only once; it terminates the recursion. The same holds for steps (S28) and (S29). Indeed, to compute $S$ in steps (S28) and (S29) in time $O(n+m)$, proceed as follows: Let $H_{1}$ be the vertices of $H$ with $r$-value 1 . If there is a vertex $h \in H$ which is adjacent to each vertex of $H_{1}$, then define $S:=\{h\}$. Otherwise, for all vertices $a \in A$, check whether $a r$-dominates $G$ using the distance matrix of $G$. If there is such a vertex $a$, then put $S:=\{a\}$; otherwise, define $S:=\{v, h\}$ for an arbitrary vertex $h$ in $H$.

Thus, all recursive steps in LOCalCRDS run in time $O(n)$, whereas those steps which terminate the recursion 
(and, hence, which are performed only once in the whole algorithm) run in linear time $O(n+m)$. With a maximal recursion depth of $O(n)$, the overall running time is $O\left(n^{2}\right)$.

The authors would like to thank their colleague Andreas Brandstädt for useful discussions. The authors were supported by DAAD (F. F. D.) and by DFG (F. N.).

\section{REFERENCES}

[1] H.-J. Bandelt and H. M. Mulder, Distance-hereditary graphs. J. Combin. Theory (B) 41 (1986) 182-208.

[2] C. Berge, Hypergraphs. North-Holland (1989).

[3] A. Brandstädt, Special graph classes - A survey. Technical Report, Gerhard-Mercator-Universität-Gesamthochschule Duisburg SM-DU-199 (1991).

[4] A. Brandstädt and F. F. Dragan, A linear-time algorithm for connected $r$-domination and Steiner tree on distancehereditary graphs. Technical Report, Gerhard-MercatorUniversität-Gesamthochschule Duisburg SM-DU-261 (1994).

[5] A. Brandstädt, V. D. Chepoi, and F. F. Dragan, The algorithmic use of hypertree structure and maximum neighbourhood orderings. Technical Report, GerhardMercator-Universität-Gesamthochschule Duisburg SMDU-244, (1994); extended abstract in Proceedings of WG'94, Lecture Notes in Computer Science 903, Springer (1995) 65-80.

[6] A. Brandstädt, F. F. Dragan, V. D. Chepoi, and V. I. Voloshin, Dually chordal graphs. Technical Report, Gerhard-Mercator-Universität-Gesamthochschule Duisburg SM-DU-225, (1993); extended abstract in Proceedings of WG'93, Lecture Notes in Computer Science 790, Springer, (1993) 237-251.

[7] A. Brandstädt, F. F. Dragan, and F. Nicolai, Homogeneously orderable graphs. Technical Report, GerhardMercator-Universität-Gesamthochschule Duisburg SMDU-271 (1994) (to appear in Theor. Comput. Sci.); extended abstract in Proceedings of WG'95, Lecture Notes in Computer Science 1017, Springer (1995) 381-395.

[8] A. Brandstädt and D. Kratsch, Domination problems on permutation and other graphs. Theor. Comput. Sci. 54 (1987) $181-198$.
[9] A. D'Atri and M. Moscarini, Distance-hereditary graphs, Steiner trees and connected domination. SIAM J. Comput. 17 (1988) 521-538.

[10] F. F. Dragan, HT-graphs: Centers, connected $r$-domination and Steiner trees. Comput. Sci. J. Moldova 1(2) (1993) 64-83.

[11] F. F. Dragan, Dominating cliques in distance-hereditary graphs. Proceedings of "Algorithm Theory-SWAT94,", 4th Scandinavian Workshop on Algorithm Theory, Aarhus, Denmark, Lecture Notes in Computer Science 824, Springer (1994) 370-381.

[12] F. F. Dragan and A. Brandstädt, $r$-Dominating cliques in graphs with hypertree structure. Proceedings of the 11 th STACS, Caen, France, Lecture Notes in Computer Science 775, Springer (1994) 735-746; Discr. Math. 162 (1996) 93-108.

[13] F. F. Dragan and F. Nicolai, $r$-Domination problems on homogeneously orderable graphs. Proceedings of Workshop on "Fundamentals of Computation Theory" FCT'95, Lecture Notes in Computer Science 965, Springer (1995) 201-210.

[14] F. F. Dragan, C. F. Prisacaru, and V. D. Chepoi, $r$-Domination and $p$-center problems on graphs (in Russian). Discr. Math. Moscow 4 (1992) 67-73.

[15] M. C. Golumbic, Algorithmic Graph Theory and Perfect Graphs. Academic Press, New York (1980).

[16] P. L. Hammer and F. Maffray, Completely separable graphs. Discr. Appl. Math. 27 (1990) 85-99.

[17] R. B. Hayward, C. Hoang, and F. Maffray, Optimizing weakly triangulated graphs. Graphs Combin. 5 (1989) 339-349.

[18] S. C. Hedetniemi and R. Laskar, Eds., Topics on domination. Ann. Discr. Math. 48 (1991).

[19] D. Kratsch, Finding dominating cliques efficiently in strongly chordal graphs and undirected path graphs. Ann. Discr. Math. 48 (1991) 225-238.

[20] D. Kratsch, P. Damaschke, and A. Lubiw, Dominating cliques in chordal graphs. Discr. Math. 128 (1994) 269275.

[21] F. Nicolai, A hypertree characterization of distance-hereditary graphs. Technical Report, Gerhard-MercatorUniversität-Gesamthochschule Duisburg SM-DU-255 (1994).

[22] R. Sritharan and J. S. Spinrad, Algorithms for weakly triangulated graphs. Discr. Appl. Math. 19 (1995) 181191. 